\title{
The Small Magellanic Cloud star cluster NGC 458. A new UBVI photometric study ${ }^{\star}$
}

\author{
G. Alcaino ${ }^{1}$, F. Alvarado ${ }^{1}$, J. Borissova ${ }^{2, \star \star}$, and R. Kurtev ${ }^{3}$ \\ ${ }^{1}$ Isaac Newton Institute of Chile, Ministerio de Educacion de Chile, Casilla 8-9, Correo 9, Santiago, Chile \\ e-mail: inewton@terra.cl \\ 2 Institute of Astronomy, Bulgarian Academy of Sciences and Isaac Newton Institute of Chile Bulgarian Branch, \\ 72 Tsarigradsko chaussèe, 1784 Sofia, Bulgaria \\ e-mail: jborisso@astro.puc.cl \\ ${ }^{3}$ Department of Astronomy, Sofia University and Isaac Newton Institute of Chile Bulgarian Branch, 1164 Sofia, Bulgaria \\ e-mail: kurtev@phys.uni-sofia.bg
}

Received 16 August 2002 / Accepted 7 January 2003

\begin{abstract}
We present a new photometry of the Small Magellanic Cloud star cluster NGC 458 in $U B V I$ filters. We determine the reddening $E(B-V)=0.04 \pm 0.02$. By comparison with the isochrones by Bertelli et al. (1994) we derive an age of $\approx 140 \mathrm{Myr}$. Three candidate blue straggler stars are proposed. The evolved "blue loop" stars of NGC 458 have masses between 4 and $5 \mathcal{M}_{\odot}$ and are hotter than model predictions.
\end{abstract}

Key words. stars: Hertzsprung-Russell (HR) and C-M diagrams - galaxies: stellar content - galaxies: Magellanic clouds galaxies: individual: $\mathrm{SMC}$ - galaxies: stars clusters

\section{Introduction}

The investigation of the intermediate-age star clusters in the Magellanic Clouds is a well-known and powerful tool to test evolutionary theories for combination of masses and metallicities not presented in our Galaxy. The NGC 458 is one of the richest clusters in the SMC. Several photometrical investigations can be found in the literature for this cluster: Arp (1959) presented the first color-magnitude diagram, and found "a large number of yellow giants, a narrow and well defined Hertzsprung gap and discontinuity of about 1 mag between the Main Sequence and those giants". The first modern CCD investigation was published by Ripepi \& Brocato in 1997. They investigated NGC 458 on NTT $B V$ images and estimated the reddening $E(B-V)=0.11$ and $110 \mathrm{Myr}$ age. The most recent photometric work on NGC 458 is the paper of Matteucci et al. (2002) where NGC 458 was investigated together with another 6 LMC and 9 SMC clusters. The authors confirm

Send offprint requests to: $\mathrm{R}$. G. Kurtev, e-mail: kurtev@phys.uni-sofia.bg

$\star$ Tables 1 and 2 are only available in electronic form at http://www . edpsciences.org

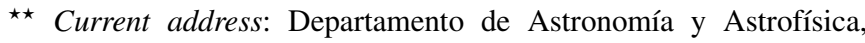
Pontificia Universidad Católica de Chile, Campus San Joaquin, Vicuña Mackenna 4860 Casilla 306 Santiago 22, Chile;

e-mail: jborisso@astro.puc.cl the morphology structure of NGC 458 - well populated Main Sequence (MS) and $\approx 30$ evolved "blue loop" stars, which are 100-150 Myr old.

All of these papers are based on $B V$ filters only. The goal of this paper is to present a new analysis of a wide region $14^{\prime} \times 14^{\prime}$ around NGC 458. We use $U B V$ and $I$ images taken with the $1.3 \mathrm{~m}$ Warsaw telescope, Las Campanas Observatory and $J H K$ photometry available in 2 MASS Second Incremental Data Release catalogue. We determine the reddening, effective temperature, luminosities and age of the cluster's stars.

\section{Observations and data reduction}

Our analysis is based on a set of "blue" $U B$ and "red" VI CCD frames obtained in October 1999 with the $1.3 \mathrm{~m}$ Warsaw telescope, Las Campanas Observatory with the $2048 \times 2048$ CCD camera. The array scale was $0.417^{\prime \prime}$ pixel $^{-1}$, giving fields of view of $14^{\prime} \times 14^{\prime}$. The stellar photometry was carried out separately for all frames using DAOPHOT/ALLSTAR (Stetson 1993). The instrumental magnitudes were transformed photometrically to the deepest reference frame in each filter. The magnitudes in the same bands of the common unsaturated stars are averaged. The instrumental values were then transformed to the standard system using 18 standard stars in the field of view from Alvarado et al. (1995) and Walker (1987). We used the "least squares" method to calculate a straight line that best fits the data for 
these standard stars. The following set of transformation equations is obtained:

$$
\begin{gathered}
B-V=0.979_{ \pm 0.013}(b-v)-0.818_{ \pm 0.019} \quad(\sigma(B-V)=0.016) \\
V-I=0.992_{ \pm 0.034}(v-i)+0.128_{ \pm 0.027} \quad(\sigma(V-I)=0.021) \\
V=0.981_{ \pm 0.007} v-0.030_{ \pm 0.026}(B-V)-4.507_{ \pm 0.023} \\
(\sigma(V)=0.039) \\
U=0.972_{ \pm 0.016} u-0.194_{ \pm 0.062}(B-V)-7.321_{ \pm 0.218} \\
(\sigma(U)=0.068)
\end{gathered}
$$

where $u, v, b-v, v-i$ are the instrumental magnitudes and colors and $U, V, B-V, V-I$ are the magnitudes and colors in the standard system. The standard error values for the coefficients are given with small digits right after each coefficient. The coefficient uncertainties correspond to the linear fits. The uncertainties of the reported sigma $-\sigma$ refer to the statistical gaussian distribution of the residuals.

Our final photometric list contains 5489 stars which have $U, B, V$ or $I$ magnitudes. The complete data set is given in Table 1, only available in the electronic form. Following the method described in Ferraro \& Paresce (1993), we calculated the rms frame-to-frame scatter of the $U, B, V$ and $I$ magnitudes. The mean rms scatter is $\approx 0.04$ mag for $U, B, V, I<20 \mathrm{mag}$ and $\approx 0.09 \mathrm{mag}$ for the fainter magnitudes. The artificial star technique (Stetson \& Harris 1988; Stetson 1991a, 1991b) was used to determine the completeness limits of the data. Our photometry is complete at $(U, B, V, I)=(19,19.5,20,20.5) \mathrm{mag}$ and to the $30^{\prime \prime}$ from the cluster center. The central $5^{\prime \prime}$ are unresolved. Comparison with $B V$ photometry of Matteucci et al. (2002) shows good agreement - the differences in colors and magnitudes are: $(B-V)=(B-V)_{M 2002}-0.02$ and $V=V_{M 2002}-0.05$, respectively. In Fig. 1 both color-magnitude diagrams constructed from the stars falling within 100" from the cluster center are overploted. The MS fiducial line and candidate variable stars are also shown (see Sects. 3 and 4 for more details).

\section{The Color-Magnitude Diagram}

To decontaminate the cluster's CMD we used the Matteucci et al. (2002) cluster limiting radius $r=100^{\prime \prime}$ encircling the most probable cluster members. This area contains cluster's stars + SMC field stars. The SMC field stars alone are selected in an annulus around the cluster with inner radius $r=6^{\prime}$ and normalized to the same area covered by the NGC 458. The $(B-V, V)$ CMDs of "cluster + field" and "field" are girded and the stars in each box in the two diagrams are counted. Then an equivalent number of stars is removed from the single boxes of the "cluster + field" CMD on the basis of the number of field stars found in the "field" CMD alone (middle panel in Fig. 2).

The $(U-V, V)$ and $(V-I, V)$ CMDs for NGC 458 stars (filled circles), decontaminated as described above, and the SMC field stars (light crosses) are presented in Fig. 3. The cluster's and the SMC field's diagrams show well defined Main Sequence stars between $17<V<22$ and $18<V<22$ mag respectively. The NGC 458 contains about 30 evolved stars with $V<17$ and $0<B-V<1 \mathrm{mag}$ - so called "blue loop"

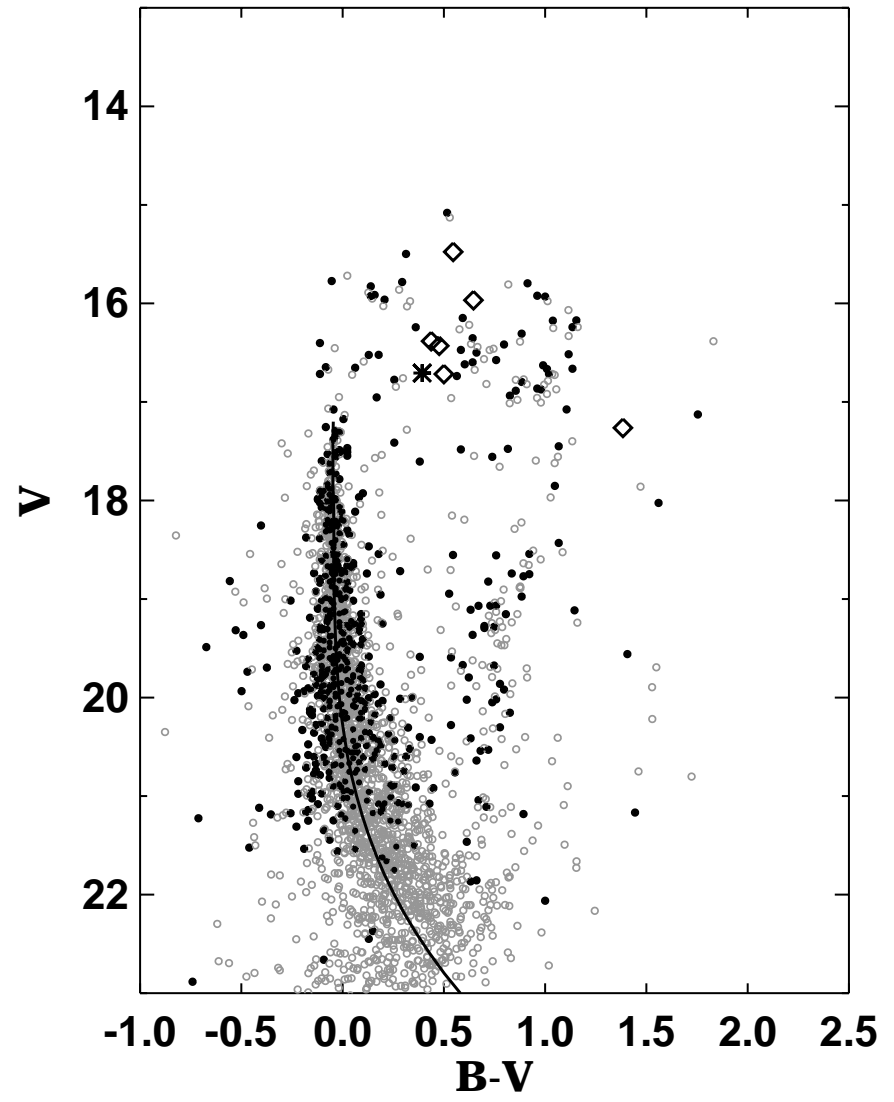

Fig. 1. The present $(B-V, V)$ color-magnitude diagram of NGC 458 (filled circle) are compared to Matteucci et al. (2002) photometry (open circle). The MS fiducial line is plotted with a solid line. The field candidate variable stars are marked by open squares, the Cepheid star No. 29 from the photometric list of Arp (1959) is plotted with an asterisk (see Sect. 4 for more details).

stars (Ripepi \& Brocato 1997; Matteucci et al. 2002). Several blue stars in the extension of the NGC 458 Main Sequence on the $(U-V, V)$ color magnitude diagram could be identified as candidates of "blue stragglers" stars (large circle in Fig. 3). The Hertzsprung gap also contains several stars, both cluster members and SMC field stars, which could be variable stars. The SMC field stars outline well defined red giant branch with $V-I>0.6$ and red clump at $V \approx 19.4 \mathrm{mag}$.

We determine the Main Sequence TO point, using the termination point of the luminosity function (corrected for completeness) of $(U-B, V),(B-V, V)$ and $(V-I, V)$ colormagnitude diagrams. The mean $V$ value was found to be at $V_{\mathrm{TO}}=17.17 \pm 0.05$ and $(U-B)_{\mathrm{TO}}=-0.30 \pm 0.08,(B-V)_{\mathrm{TO}}=$ $-0.05 \pm 0.04,(V-I)_{\mathrm{TO}}=-0.05 \pm 0.05 \mathrm{mag}$. The errors associated with the measurements include the photometry errors and the quoted in Sect. $2 \sigma$ values of the transformation to the standard system. The mean ridgeline of the NGC 458 Main Sequence stars was determined following the polynomial fitting technique by Sarajedini (1994). For the stars fainter than $V=20 \mathrm{mag}$ was added the photometry of Matteucci et al. (2002). The error of the polynomial fit is 0.08 mag. In Fig. 1 the mean $(B-V, V)$ ridgeline is overploted as a solid line.

To determine the reddening of NGC 458 we used the best fit of $(B-V)$ vs. $(U-B),(V-I)$ vs. $(B-V)$, and $(V-I)$ vs. 


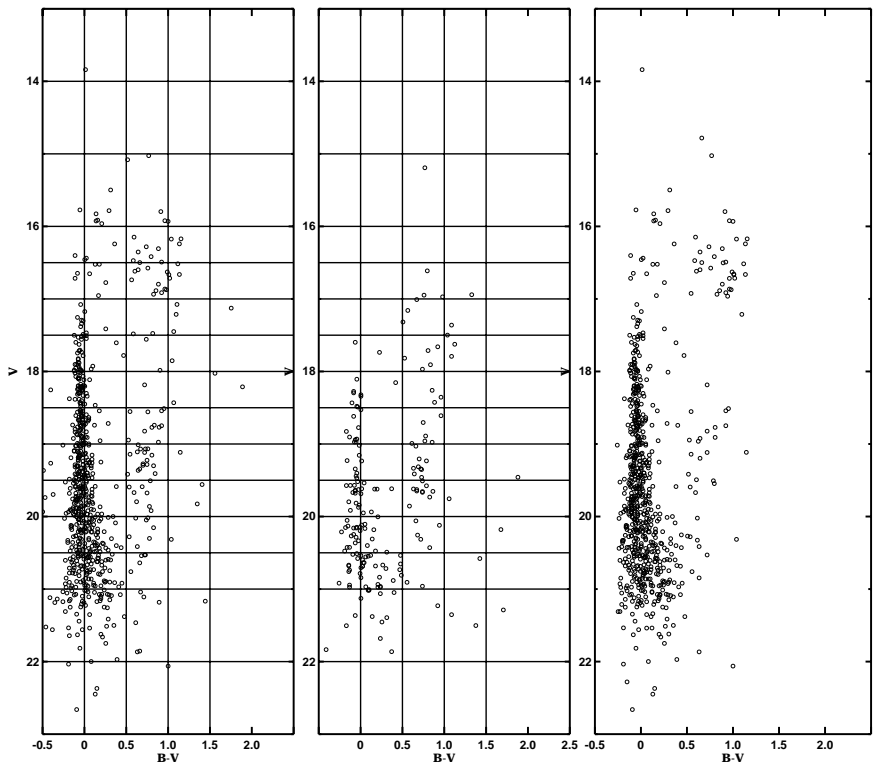

Fig. 2. The $(B-V, V)$ color-magnitude diagram for NGC $458+$ field stars within $100^{\prime \prime}$ (left panel). The field CMD (middle panel) is based on all stars in the normalized area with $r \geq 6^{\prime}$ (see text). On the righthand-side panel the decontaminated "cluster" CMD is presented.

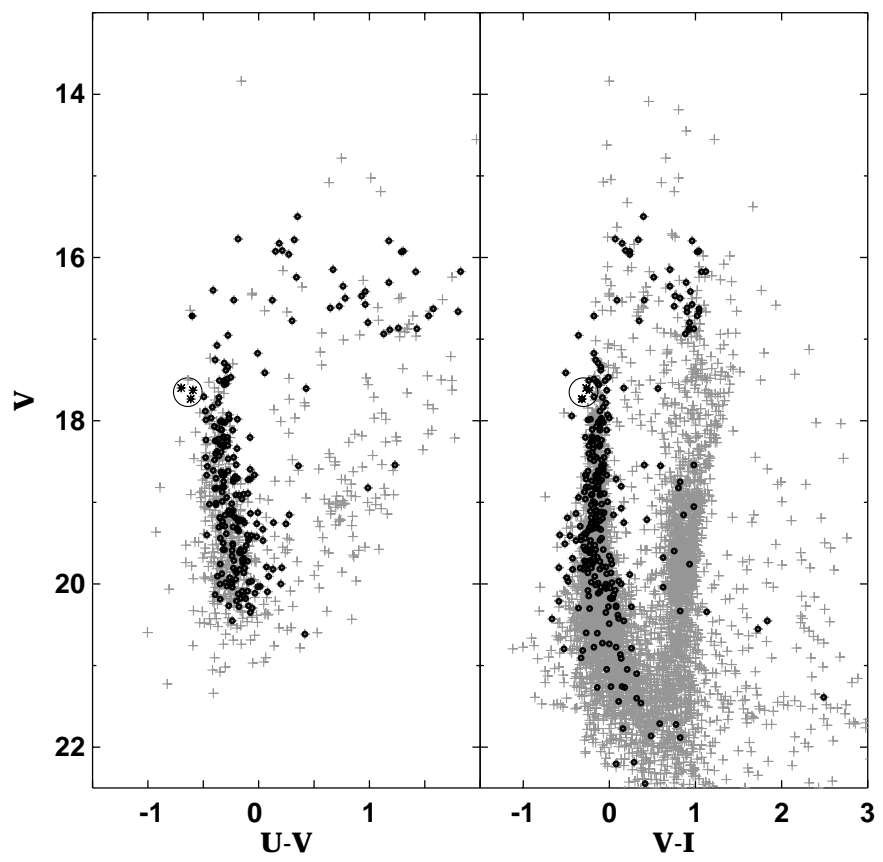

Fig. 3. The $(U-V, V)$ and $(V-I, V)$ CMDs for SMC field stars (light crosses) and NGC 458 stars (filled circles). The large circle indicates the BSS area, the three candidate BSS stars are plotted by asterisks (see Sect. 4 for more details).

$(U-B)$ distributions for Main Sequence stars to the color-color lines from Bessell et al. (1998). The resulting unweighted mean reddening is $E(B-V)=0.04 \pm 0.02$. The error indicates the standard deviation of the mean value. The value obtained by this manner is smaller than those $(E(B-V)=0.11)$ reported in Ripepi \& Brocato (1997). On the other hand our reddening value is the same, within the errors, with those given in Arp (1959) and the mean reddening value $E(B-V)=0.037$ for SMC from Schlegel et al. (1998). Using the above determined reddening, the commonly accepted for SMC $Z=0.004$ and $(m-M)_{0}=18.85$, we fitted our data with isochrones from Padua's library (Bertelli et al. 1994). The Main Sequence termination point is very well defined in the $(B-V, U),(U-B, U)$ and $(V-I, U)$ CMDs. The best fit was obtained for $142 \pm 30 \mathrm{Myr}$. The error is computed by changing the reddening from 0.0 to 0.06 and the distance modulus from 18.85 to 19.10 (see the next paragraph). This result is in the end of the age interval given in Matteucci et al. (2002), who estimate the age of NGC 458 between 100 and 150 Myr.

The field stars in the NGC 458 region present a well defined red giant clump. We used the method suggested by Girardi \& Salaris (2001) to evaluate the SMC distance. The red clump is determined at $V=19.44 \pm 0.04 \mathrm{mag}$ and $I=18.67 \pm 0.04$. The calculated distance $\mu=19.11 \pm 0.2$ is larger than this in Girardi \& Salaris (2001), but is very close to those obtained by Massey et al. (1995) $19.10 \pm 0.3$ from spectroscopic parallaxes.

\section{Evolved stars}

The location of blue and red supergiants on the colormagnitude diagram and accurate determination of their temperatures and luminosities can help us to verify the predictions of stellar evolutionary models at low metallicity. On the statistically decontaminated color-magnitude diagrams of NGC 458 (Figs. 1 and 3) we can identify thirty nine evolved "blue loop" stars. There is a cross-identification for thirty of them and $J H K$ photometry given in 2 MASS Second Incremental Data Release Catalogue. Their optical and near IR magnitudes are given in Table 2 (available in the electronic form) and Fig. 4 shows $(J-K, K)$ (panel a) and $(V-K, J-K)$ (panel b) colormagnitude and color-color diagrams. The 2 MASS magnitudes of NGC 458 are transformed to the AAO system using color transformations given in the description of the 2 MASS catalogue. The blue loop stars of NGC 458 are located in the $(J-K)$ color interval between 0 and 1 , the brightest star in NGC 458 stands at $K=13.4 \pm 0.05$. On the dereddened $\left((V-K)_{0},(J-K)_{0}\right)$ color-color diagram we overploted the predicted locus for $\log g=+0.5$ from Table 2 of Bessell et al. (1998) for ATLAS9 overshoot models. There are no departures from this relation, except for three stars around $(V-K)_{0}=2$ and $(J-K)_{0}=0$, marked with crosses. Careful examination of these stars show, that they lie on the extension of the MS.

Our next step was to transform the dereddened $V-K$ color to the effective temperature and $K$ magnitudes to the luminosities through the tables of Bessell et al. (1998). We adopted the distance modulus for SMC 18.9 and $E(B-V)=0.04$. The resultant $\mathrm{H}-\mathrm{R}$ diagram is plotted on the Fig. 4 panels $\mathrm{c}$ and d. In Fig. 4, panel c, the evolutionary tracks from Lejeune $\&$ Schaerer (2001) for $Z=0.004$ for 4 and $5 \mathcal{M}_{\odot}$ are overploted, while on panel d we used the isochrones by Bertelli et al. (1994). The two sets of models possess the same input physics, but differ in the degree of convective core overshoot and envelope undershot. In our case both models match well to the observational data. The plots show that NGC 458 brightest stars have masses between 4 and $5 \mathcal{M}_{\odot}$ and effective temperatures $\log \left(T_{\text {eff }}\right)$ between 3.65 and 3.9. The main source of errors 

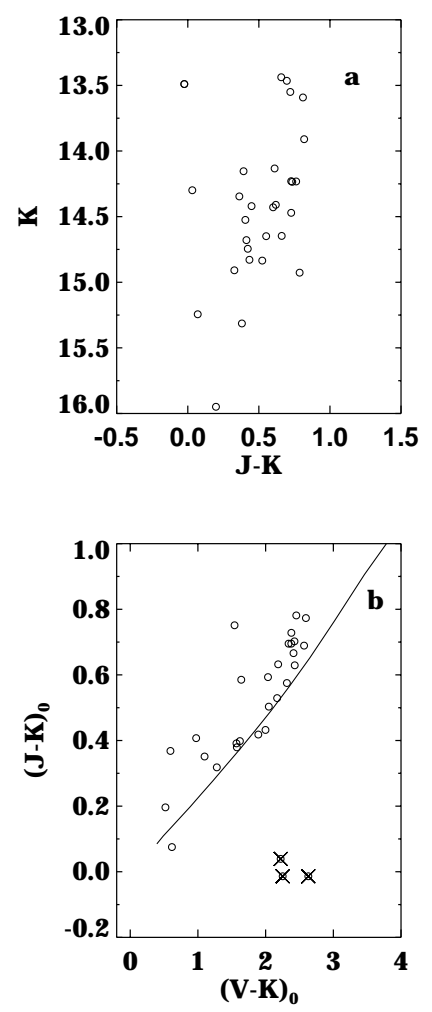

G. Alcaino et al.: The Small Magellanic Cloud star cluster NGC 458
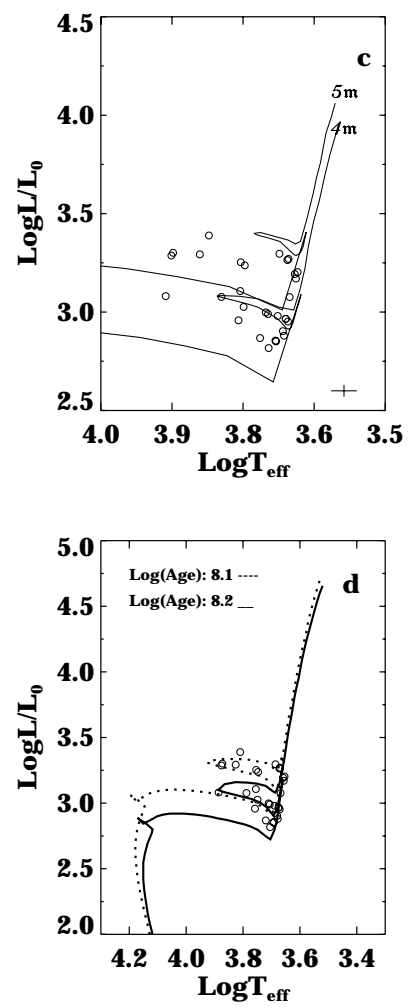

Fig. 4. The $J-K, K$ (panel a)), $V-K, J-K$ (panel b)) color magnitude and color-color diagrams of NGC 458. On panel c) on the H-R diagram the evolutionary tracks from Lejeune \& Schaerer (2001) for $Z=0.004$ and 4 and $5 M_{\odot}$ are overploted, while on panel d) we used the isochrones by Bertelli et al. (1994). Typical error bars are shown in panel c).

here is the uncertainty of the reddening determination and the typical error bars are shown on panel c. Keller (1999) determined for the "blue loop" stars of NGC 458 lower temperatures and much higher luminosities. One possible explanation is that Keller used rather old $B, V$ photometry of Arp (1959) instead of $J K$ photometry. Nevertheless our values also confirm Keller's suggestion of the mass dependence of the mixing-length parameter $\alpha_{P}$. And finally, as can be seen in Fig. 4 (panel d), the "blue loop" stars match well isochrones $\log ($ Age $)=8.1$ and $\log ($ Age $)=8.2$. This is in agreement with the previous result of 142 Myr obtained by using the MS termination point.

Since our observational material is obtained on three nights (12, 13 and 16.10.1999) we can perform a search for undetected variables by comparison of the brightness variations. We determined the mean magnitudes and their standard deviations for the entire sample of stars. Naturally, the variables are expected to show much larger brightness variations than the non-variable stars in the same magnitude interval. The five sigma criterion selected eight candidate variable stars. On the $(B-V, V)$ (Fig. 2) color magnitude diagram they are located in the so called Hertzsprung gap. One of them is No. 29 from the photometric list of Arp (1959). He classified it as a Cepheid. Unfortunately, this is the only candidate in our list which is a probable cluster member, the remaining ones are field stars. Taking into account their position on the color-magnitude diagram most of them can be classified also as Cepheid variables.
Table 3. Candidate BSS stars.

\begin{tabular}{cccccc}
\hline \hline$X$ & $Y$ & $r$ & $U-B$ & $B-V$ & $V$ \\
\hline 993.20 & 1148.02 & 0.049 & -0.597 & -0.103 & 17.599 \\
955.90 & 1162.26 & 0.318 & -0.578 & -0.035 & 17.734 \\
887.70 & 1188.67 & 0.824 & -0.530 & -0.064 & 17.627 \\
\hline
\end{tabular}

One is a probable red variable. Our observational material does not allow us to obtain the periods and light curves of the suspected variables.

On the basis of their location on the $(U-V, U),(U-B, U)$ and $(U-V, V)$ statistically decontaminated CMDs, we identified three candidate BSS (plotted by asterisks in Fig. 3). The $X$ and $Y$ coordinates (in pixels), distance from the cluster center (in arcmin) and $U-B, B-V$ colors and $V$ magnitudes for these candidate BSS are listed in Table 3. Careful examinations were made to ensure that the internal errors of the BSS candidates are the same as those of the MS stars at the same brightness level. All three star lie within 0.8 arcmin from the cluster center. For comparison 8 BSS candidates in NGC 330 were also found (Keller et al. 2000).

\section{Summary}

We presented a new photometric analysis of NGC 458 in $U B V$ and $I$ filters. Comparison with previous $B V$ photometry of Matteucci et al. (2002) shows good agreement and in this paper we reported the first UI magnitudes of the cluster stars. We confirmed the overall color-magnitude morphology of NGC 458 outlined by Ripepi \& Brocato (1997) and Matteucci et al. (2002). On the basis of the location on the $(U-V, U),(U-B, U)$ and $(B-V, V)$ CMDs, we identified three candidate blue straggler stars. We determined reddening $E(B-V)=0.04 \pm 0.02$. The best fit of the isochrones by Bertelli et al. (1994) is for an age of $\approx 140 \mathrm{Myr}$, which is in the upper limit of the Matteucci et al. (2002) age interval. Cross - identification of the optical magnitudes of the "blue loop" stars with near IR photometry available in the 2 MASS catalogue allows us to transform the $V-K$ color to the effective temperature and $K$ magnitudes to the luminosities. The brightest evolved stars in NGC 458 have masses between 4 and $5 \mathcal{M}_{\odot}$ and are hotter than the model predictions.

Acknowledgements. J.B. and R.K. would like to thank Ch. Chavdarov for his help. This publication makes use of data products from the Two Micron All Sky Survey, which is a joint project of the University of Massachusetts and the Infrared Processing and Analysis Center/California Institute of Technology, funded by the National Aeronautics and Space Administration and the National Science Foundation. The authors gratefully acknowledge the useful comments by an anonymous referee.

\section{References}

Arp, H. 1959, AJ, 64, 175

Alvarado, F., Wenderoth, E., Alcaino, G., \& Liller, W. 1995, AJ, 110, 646

Bessell, M. S., Castelli, F., \& Plez, B. 1998, A\&A, 333, 231 
Bertelli, G., Bressan, A., Chiosi, C., Fagotto, F., \& Nasi, E. 1994, A\&AS, 106, 275

Ferraro, F. R., \& Paresce, F. 1993, AJ, 106, 154

Girardi, L., \& Salaris, M. 2001, MNRAS, 323, 109

Keller, S. C. 1999 , AJ, 118, 889

Keller, S. C., Bessell, M. S., \& Da Costa, G. S. 2000, AJ, 119, 1748

Matteucci, A., Ripepi, V., Brocato, E., \& Castellani, V. 2002, A\&A, 387,861

Massey, P., Lang, C., De Gioia-Eastwood, K., \& Garmany, C. 1995, ApJ, 438, 188

Ripepi, V., \& Brocato, E. 1997, in Advances in Stellar Evolution, Proc. of the Workshop Stellar Ecology, held in Marciana Marina, Elba, Italy, 23-29 June 1996 (Cambridge: Cambridge University Press), 70
Sarajedini, A. 1994, AJ, 107, 618

Schlegel, D. J., Finkbeiner, D. P., \& Davis, M. 1998, ApJ, 500, 525 Lejeune, T., \& Schaerer, D. 2001, A\&A, 365, 535

Stetson, P. B. 1991a, in The Formation and Evolution of Star Clusters, ed. K. A. Janes (San Francisco: ASP), ASP Conf. Ser., 13, 88

Stetson, P. B. 1991b, in Precision Photometry: Astrophysics of the Galaxy, ed. A. G. D. Philip, A. R. Upgren, \& K. A. Janes (Schenectady: L. Davis Press), 69

Stetson, P. B. 1993, User's Manual for DAOРнOT II

Stetson, P. B., \& Harris, W. E. 1988, AJ, 96, 909

Walker, M. 1987, PASP, 99, 179 